\title{
Cognitive status in patients with multiple sclerosis in Lanzarote
}

\author{
This article was published in the following Dove Press journal: \\ Neuropsychiatric Disease and Treatment \\ 30 June 2016 \\ Number of times this article has been viewed
}

\section{María Yaiza Pérez-Martín' \\ Pablo Eguia-del Río \\ Montserrat González-Platas' \\ Alejandro Jiménez-Sosa ${ }^{3}$ \\ 'Service of Neurology, Complejo \\ Hospitalario Universitario de \\ Canarias, La Laguna, ${ }^{2}$ Service of \\ Neurology, Doctor José Molina \\ Orosa Hospital, Arrecife, Lanzarote, \\ ${ }^{3}$ Unit of Research, Complejo \\ Hospitalario Universitario de \\ Canarias, La Laguna, Spain}

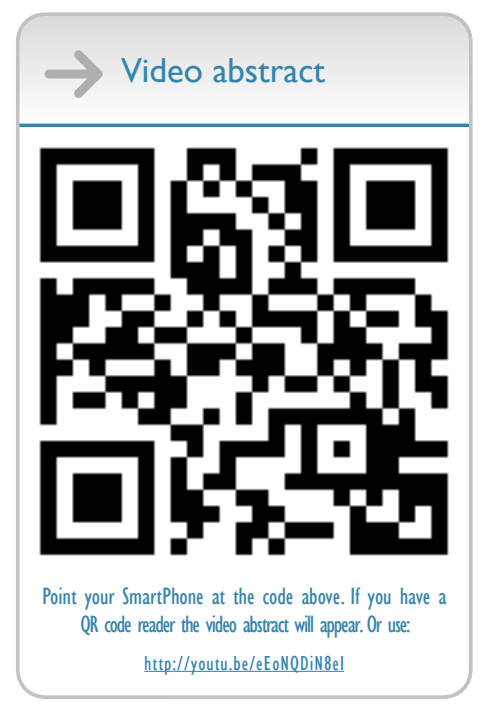

Correspondence: María Yaiza Pérez-Martín Servicio de Neurología, Edificio de Actividades Ambulatorias, Planta $3^{\mathrm{a}}$, Complejo Hospitalario Universitario de Canarias, Ofra s/n, La Laguna, Santa Cruz de Tenerife 38320, España

Tel +34616464403

Fax +34922 31 927।

Email yaizaneuro@hotmail.com
Objectives: Cognitive impairment is a common feature in multiple sclerosis affecting $43 \%-72 \%$ of patients, which involves cognitive functions such as memory, processing speed, attention, and executive function. The aim of this study was to describe the extent and pattern of the involvement of cognitive impairment and psychological status in all patients with multiple sclerosis on a small Spanish island.

Patients and methods: In all, 70 patients and 56 healthy controls were included in the study between February 2013 and May 2013. All participants were assessed using the Brief Repeatable Battery of Neuropsychological Test. The patients also completed instruments to evaluate the presence of fatigue, perceived cognitive dysfunction, and symptoms of anxiety and depression. All procedures were performed in a single session.

Results: Cognitive impairment, defined as a score $<1.5$ standard deviation on two subtests of the battery, was present in $35 \%$ of the participants. The most frequently affected domain was working memory, followed by verbal memory and processing speed. Disease duration showed a moderate correlation with visuospatial memory and processing speed. The Expanded Disability Status Scale score correlated with verbal and processing speed. Verbal memory was correlated with depression symptoms and fatigue.

Conclusion: Cognitive impairment was present in $35 \%$ of the study population. The most affected domains were working memory and verbal memory. Working memory and verbal fluency deficit are independent factors of disease evolution. Cognitive decline is related to clinical variables and psychological measures such as fatigue or depression but not to anxiety.

Keywords: cognitive status, cognitive impairment, Lanzarote, multiple sclerosis

\section{Introduction}

Neurodegenerative conditions, such as dementia, multiple sclerosis (MS), Parkinson's disease, and Huntington's disease, are often characterized by significant neuropsychological deficits that interfere significantly with patient's abilities and quality of life. ${ }^{1-4}$

Cognitive impairment is a common feature in MS affecting $\sim 43 \%-72 \%$ of patients ${ }^{5,6}$ and is characterized as other signs and symptoms of the disease by variability and heterogeneity. However, dementia is rare, and the more common clinical presentation is one of the specific and subtle cognitive deficits. ${ }^{7}$

The most frequently affected cognitive functions are attention, speed of information processing, memory, executive functions, and visuospatial abilities, ${ }^{8}$ although new domains such as theory of mind have also been identified. ${ }^{9}$ Involvement of cognitive functions such as language, praxis, and gnosis is rare and less well studied.

The influence of the duration of the illness on cognitive functioning is still a matter of controversy. As regards clinical presentation, the progressive forms of MS 
(both secondary progressive MS and primary progressive MS) are more involved in both degree and altered domains as compared to relapsing-remitting multiple sclerosis (RRMS). ${ }^{10,11}$

Some authors point out that impairments are almost invariably a complication of the later stages of the disease when there is axonal loss with the involvement of large areas of white matter, disconnection between several cortical areas of association, and disconnection between cortical and subcortical areas, but impaired cognitive function appears to be present even in the early stages of the disease. Studies with long-term follow-up indicate that cognitive function decreases as the disease progresses. ${ }^{12,13}$ The degree of neurological impairment is shown as a predictor of cognitive decline in the follow-up studies. ${ }^{14}$

In the case of fatigue, this can interfere negatively, especially in tasks that require sustained mental effort such as working memory tests or sustained attention. ${ }^{15}$

Neuropsychological dysfunction severely affects the patients' lives ${ }^{16}$ in terms of their ability to keep their jobs, ${ }^{17}$ and they require greater assistance with daily living activities. Cognitively compromised patients are also more likely to have problems with socialization than cognitively intact MS patients. ${ }^{18}$ Cognitive impairment has been associated with nonsomatic symptoms of depression in RRMS, and this finding supports the importance of evaluating depressive symptoms when cognitive impairment is suspected in patients with RRMS. ${ }^{19}$ Anxiety has been less studied than depression in MS patients, although there is evidence that high levels of anxiety are associated with poor performance in cognitive tasks, especially processing speed, working memory, and visual-spatial memory. ${ }^{20,21}$
This study was conducted in Lanzarote, the easternmost landmass of the Canary Islands, Spain (Figure 1). The only study of the prevalence of MS on this island was published in 1980s, which reported a low prevalence of $15 / 100,000 .{ }^{22}$ The population of the island at that time was 60,000 inhabitants, and the authors concluded that the rate was higher than expected because of the geographical situation of the island. More recently, a study presented at the LXV Annual Meeting of the Spanish Society of Neurology identified a total of 70 patients with MS on the island. This figure gives a prevalence of 49/100,000. ${ }^{23}$

Other epidemiological studies conducted in the region have found that the Canary Islands is a medium-to-high risk area like Spain and other Mediterranean countries. ${ }^{24,25}$ Thus, the Canary Islands, despite being closer to the equator, are still a medium risk area.

The aim of this study was to describe the extent and pattern of the involvement of cognitive impairment and the psychological status in all patients of a MS cohort. A study of the full MS population, which rules out a possible selection bias, should provide a more comprehensive view of the cognitive impairment spectrum in MS.

\section{Patients and methods}

The study protocol was approved by the Ethics Committee for Clinical Research of the University Hospital of the Canary Islands and the Medical Director of Hospital Doctor José Molina Orosa. The study was conducted in accordance with the Declaration of Helsinki. ${ }^{26}$ All patients signed written informed consent. The study was performed between February and May 2013.

\section{Participants}

Seventy patients with MS or clinically isolated syndrome (CIS) treated at the Doctor José Molina Orosa Hospital in

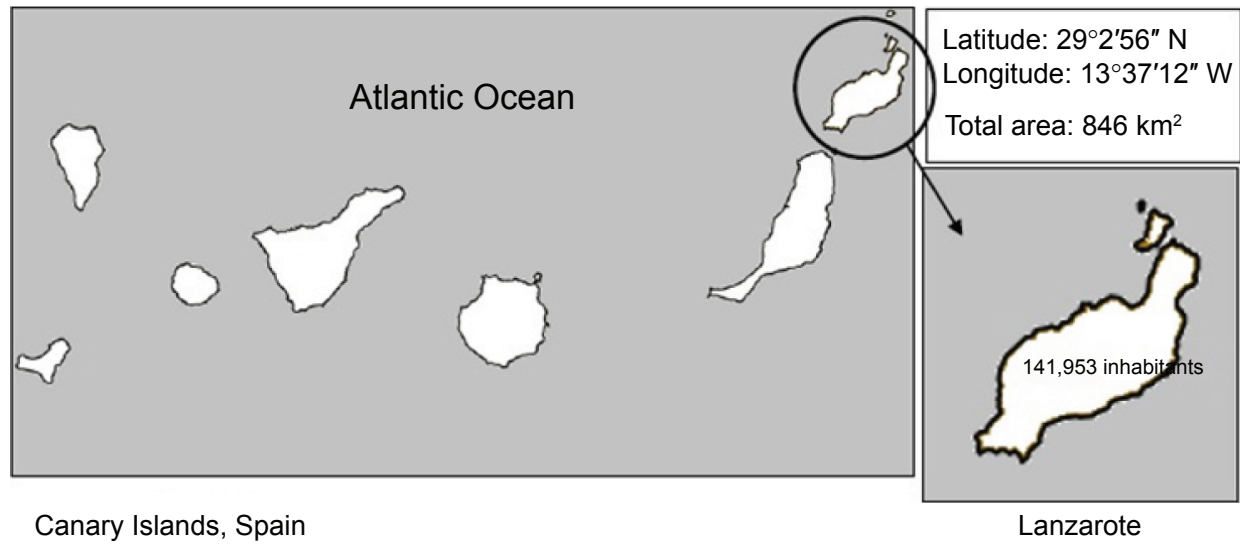

Figure I Study area.

Abbreviations: $\mathrm{N}$, north; $\mathrm{W}$, west. 
Lanzarote (the hospital sees all the MS or CIS patients on the island) and 56 healthy controls (HCs) matched by sex, age, and schooling were included in the study.

The inclusion criteria for the patients were as follows:

- willing and able to sign written informed consent;

- MS diagnosed according to the McDonald criteria $2005^{27}$ or CIS; and

- neurologically stable MS (with no evidence of relapse or steroids treatment in the last 4 weeks preceding the enrollment).

The inclusion criterion for the control group (CG) was willing and able to sign the written informed consent.

The exclusion criteria for the CG were as follows:

- no history of psychiatric or neurological disorders and

- no history of drug abuse or any major medical illness.

Six patients did not participate in the study: three patients were not on the island at the time, one patient could not attend due to severe physical disability, and two patients refused to participate in the study.

\section{Measurement instruments}

The Spanish version of the Brief Repeatable Battery of Neuropsychological Test (BRB-N) ${ }^{6}$ is used to assess the cognitive status of all the participants. The BRB-N includes the following tests:

- Selective Reminding Test (SRT) ${ }^{28}$ as a measure of verbal learning and delayed recall. This test gives three different scores: SRT-Long-Term Storage (SRT-LTS), SRT-Consistent Long-Term Retrieval (SRT-CLTR), and SRT-Delayed (SRT-D).

- 10/36 Spatial Recall Test (SPART), an extended version of the original form the 7/24 SPART to assess visuospatial learning and delayed recall. ${ }^{29}$ This test gives two scores: SPART-Total and SPART-Delayed (SPART-D).

- Symbol Digit Modalities Test (SDMT) ${ }^{30}$ as a measure of complex attention and processing speed.

- Paced Auditory Serial Addition Test (PASAT) ${ }^{31}$ as a measure of sustained attention and working memory.

- Controlled Oral Word Association Test ${ }^{32}$ as a measure of verbal fluency.

The Multiple Sclerosis Neuropsychological Questionnaire (MSNQ), ${ }^{33}$ the Hospital Anxiety and Depression Scale, ${ }^{34}$ and the Fatigue Severity Scale ${ }^{35}$ were administered to the patients.

\section{Procedure}

All evaluations were performed by a neuropsychologist who was an expert in MS and familiar with the administration of all tests. These tests were administered in a single session of $\sim 1$ hour.

A $z$ score (direct score mean/standard deviation [SD]) was created for each cognitive domain according to standard practice to obtain a global cognitive-performance score. The different subtests were weighted by their $z$ score to balance the tasks.

Verbal memory $=\frac{z \text {-LTS }+z-\mathrm{CLTR}+z-\mathrm{SRT}-\mathrm{D}}{3}$

$\underset{\text { memory }}{\text { Visuospatial }}=\frac{z \text {-SPART total }+z \text {-SPART-D }}{2}$

$\underset{\text { speed }}{\text { Processing }}=z$-total correct responses of SDMT

$\begin{aligned} & \text { Working } \\ & \text { memory }\end{aligned}=\frac{z \text {-total correct responses of PASAT 3" + PASAT 2" }}{2}$

$\begin{aligned} & \text { Verbal } \\ & \text { fluency }\end{aligned}=\frac{z \text {-semantic }+z \text {-phonetic total correct responses }}{2}$

Finally, a global cognitive function score ( $z$-Global) was obtained by calculating the mean of the $z$ scores from the five cognitive domains.

\section{Cognitive impairment definition}

Different cognitive impairment criteria were used: $<1.0$ $\mathrm{SD},<1.5 \mathrm{SD}$, and $<2.0 \mathrm{SD}$ (compared to the $\mathrm{CG}$ ) in one, two, or three subtests of the battery, respectively.

Interviews were also conducted with family members about their level of functionality (food preparation, medication management, use of money, travel outside home) in the case of patients with suspected dementia.

Dementia was diagnosed following the fourth edition of the Diagnostic and Statistical Manual of Mental Disorders, text revised (DSM-IV-TR). ${ }^{36}$

\section{Statistical analysis}

Categorical variables were expressed as frequencies and percentages. Continuous variables were expressed as mean $\pm \mathrm{SD}$. Normality was tested using the KolmogorovSmirnov test. All the variables analyzed followed a normal distribution except PASAT 3", and the scores were log transformed before analysis of covariance. 
Table I Clinical and demographic characteristics of both $\mathrm{HC}$ and MS patients

\begin{tabular}{llll}
\hline & $\begin{array}{l}\text { HC } \\
(\mathbf{n}=\mathbf{5 6})\end{array}$ & $\begin{array}{l}\text { MS patients } \\
(\mathbf{n}=\mathbf{6 0})\end{array}$ & P-value \\
\hline Sex (female), n (\%) & $40(7 \mathrm{I} .4)$ & $36(60)$ & 0.19 \\
Age (years) & $42.1 \pm 9.38$ & $43.9 \pm \mathrm{I} 1.54$ & 0.36 \\
Schooling (years) & $1 \mathrm{I} .2 \pm 2.87$ & $\mathrm{II} .5 \pm 3.10$ & 0.93 \\
Educational level, n (\%) & & & \\
Primary & $22(39.3)$ & $22(36.7)$ & \\
Secondary & $19(33.9)$ & $20(33.4)$ & \\
University & $15(26.8)$ & $18(30.1)$ & \\
MS course, n (\%) & & & \\
RR & & $49(81.7)$ & \\
SP & & $5(8.3)$ & \\
PP & & $4(6.7)$ & \\
CIS & & $2(3.3)$ & \\
Disease duration (years) & & $13.5 \pm 10.9$ & \\
EDSS & & $2.4 \pm 2.13$ & \\
\hline
\end{tabular}

Note: Data presented as mean \pm standard deviation unless otherwise stated.

Abbreviations: $\mathrm{HC}$, healthy control; MS, multiple sclerosis; RR, relapsing remitting; $\mathrm{SP}$, secondary progressive; PP, primary progressive; CIS, clinically isolated syndrome; EDSS, Expanded Disability Status Scale.

Analysis of covariance was performed, including cognitive domains such as dependent variables and controlling for age and schooling.

Correlation between the cognitive domains and the patient's clinical variables and questionnaires were assessed using the Pearson's correlation test.

Statistical analysis was performed with SPSS v20.0 software (IBM Corporation, Armonk, NY, USA). $P$-values were corrected for multiple testing problem using the Bonferroni correction method $(\alpha / k: 0.05 / 6=0.008)$, considering $P$-values $<0.008$ as statistically significant.

\section{Results}

Four patients met the diagnostic criteria for dementia. These patients were excluded from the analysis.
Table 1 shows the clinical and demographical characteristics of $\mathrm{HC}(\mathrm{n}=56)$ and patients (MS; $\mathrm{n}=60$; both groups had similar distributions of sex, age, and schooling).

The patients scored less than controls, in the controlling for age and schooling, on all variables of the BRB-N except visuospatial memory (Table 2).

A high prevalence of cognitive impairment $(75.0 \%)$ was obtained when using a lenient criterion $(<1.0 \mathrm{SD}$ in one subtest). The prevalence was $35.0 \%$ with a moderately stringent criterion ( $<1.5 \mathrm{SD}$ in two subtests), and the prevalence was $16.6 \%$ when using the most stringent criterion of cognitive impairment ( $<2 \mathrm{SD}$ in three tests; Table 3$)$. These percentages include patients with dementia.

The most frequently affected domain was working memory with an impairment in $43.8 \%$ of the patients (28 patients), followed by verbal memory in $21.7 \%$ (13 patients), processing speed in $15.0 \%$ (nine patients), verbal fluency in $13.3 \%$ (eight patients), and finally, visuospatial memory in $11.7 \%$ (seven patients). Nine patients had a lower global impairment index.

When clinical variables were analyzed, disease duration had a moderate correlation with visuospatial memory and processing speed $(r=-0.400$ and $r=-0.370, P<0.008)$. The Expanded Disability Status Scale (EDSS) score showed correlations with verbal memory, processing speed, and global cognitive index ( $r=-0.344$ to $-0.395, P<0.008$; Table 4).

The questionnaire scores are listed in Table 5. Symptoms of anxiety were present in $18.3 \%$ of patients, and depression symptoms were present in $33.3 \%$ of patients. Fatigue (scores $>4.1$ ) was present in 22 patients. MSNQ score and anxiety symptoms were not associated with performance in any of the cognitive domains. Verbal memory correlated with

Table 2 Cognitive measures of both $\mathrm{HC}$ and MS patients (controlling by age and schooling)

\begin{tabular}{|c|c|c|c|c|c|c|}
\hline & $H C(n=56)$ & K-S (P-value) & MS patients $(n=60)$ & K-S (P-value) & $\boldsymbol{F}$ & P-value \\
\hline \multicolumn{7}{|l|}{ SRT } \\
\hline LTS & $43.6 \pm 15.48$ & 0.49 & $32.1 \pm 16.43$ & 0.98 & 15.69 & $<0.001$ \\
\hline CLTR & $35.6 \pm 17.00$ & 0.98 & $24.8 \pm 16.98$ & 0.77 & 11.27 & 0.001 \\
\hline SRT-D & $8.8 \pm 2.91$ & 0.11 & $5.9 \pm 2.97$ & 0.28 & 31.69 & $<0.001$ \\
\hline \multicolumn{7}{|l|}{ I0/36 SPART } \\
\hline SPART-Total & $21.1 \pm 4.83$ & 0.31 & $20.3 \pm 5.87$ & 0.61 & 0.22 & 0.64 \\
\hline SPART-D & $7.3 \pm 2.18$ & 0.17 & $7.5 \pm 2.38$ & 0.06 & 0.53 & 0.46 \\
\hline SDMT & $52.4 \pm \mid 3.51$ & 0.27 & $46.6 \pm 14.74$ & 0.68 & 5.02 & 0.02 \\
\hline \multicolumn{7}{|l|}{ PASAT } \\
\hline PASAT 3"-correct & $40.5 \pm 14.09$ & $0.40^{\mathrm{a}}$ & $29.2 \pm 17.56$ & 0.24 & 14.50 & $<0.001$ \\
\hline COWAT & $36.2 \pm 11.15$ & 0.08 & $31.7 \pm 11.18$ & 0.62 & 4.47 & 0.03 \\
\hline Semantic fluency (animals) & $21.8 \pm 4.45$ & 0.22 & $19.4 \pm 5.16$ & 0.52 & 6.78 & 0.01 \\
\hline
\end{tabular}

Notes: Data are expressed as mean \pm standard deviation. aPASAT 3"-correct scores were log transformed before ANCOVA analysis.

Abbreviations: HC, healthy control; MS, multiple sclerosis; K-S, Kolmogorov-Smirnov test; SRT, Selective Reminding Test; LTS, Long-Term Storage; CLTR, Consistent Long-Term Retrieval; SRT-D, SRT-Delayed; SPART, Spatial Recall Test; SPART-D, SPART-Delayed; SDMT, Symbol Digit Modalities Test; PASAT, Paced Auditory Serial Addition Test; COWAT, Controlled Oral Word Association Test; ANCOVA, analysis of covariance. 
Table 3 BRB-N results in patients according to different strength criteria

\begin{tabular}{|c|c|c|c|}
\hline \multirow[t]{2}{*}{ MS patients $(n=60)$} & \multicolumn{3}{|l|}{ Criteria } \\
\hline & $\leq \mathrm{I} .0 \mathrm{SD}$ & $\leq \mathrm{I} .5 \mathrm{SD}$ & $\leq 2.0 \mathrm{SD}$ \\
\hline \multicolumn{4}{|l|}{ Abnormal subtest, n (\%) } \\
\hline$\geq$ I subtest & $45(75.0)$ & $39(65.0)$ & $36(60.0)$ \\
\hline$\geq 2$ subtest & $30(50.0)$ & $21(35.0)$ & $12(20.0)$ \\
\hline$\geq 3$ subtest & $23(38.3)$ & II (I8.3) & $10(16.6)$ \\
\hline
\end{tabular}

Abbreviations: BRB-N, Brief Repeatable Battery of Neuropsychological Test; MS, multiple sclerosis; SD, standard deviation.

symptoms of depression $(r=-0.415, P=0.001)$ and fatigue ( $r=-0.383, P=0.003$ ).

MSNQ correlated with depression $(r=-0.405, P=0.001)$ and anxiety $(r=-0.255, P=0.049)$.

\section{Discussion}

The aim of this study was to describe the extent and pattern of cognitive impairment in all the population affected by MS on a small island located in the southwest of Europe. Lanzarote is an interesting place for MS study because its geographical location confers a high exposure to UV radiation for most of the year and patients probably have an optimal vitamin $\mathrm{D}$ serum level. Low vitamin D status is a possible environmental risk factor for MS development and outcome..$^{37,38}$

Table 4 Correlations between cognitive domains, clinical variables, and questionnaire results

\begin{tabular}{|c|c|c|c|c|c|c|}
\hline & VM & VSM & PS & WM & VF & GCS \\
\hline \multicolumn{7}{|c|}{ Disease duration } \\
\hline$r$ & -0.210 & -0.400 & -0.370 & -0.074 & -0.312 & -0.330 \\
\hline$P$ & 0.114 & $0.002 *$ & $0.005^{*}$ & 0.579 & $0.017^{*}$ & $0.012^{*}$ \\
\hline \multicolumn{7}{|c|}{ EDSS score } \\
\hline$r$ & -0.344 & -0.296 & -0.395 & -0.151 & -0.262 & -0.355 \\
\hline$P$ & $0.008^{*}$ & $0.023^{*}$ & $0.002^{*}$ & 0.253 & $0.045^{*}$ & $0.006 *$ \\
\hline \multicolumn{7}{|c|}{ Anxiety score } \\
\hline$r$ & -0.062 & 0.066 & 0.086 & -0.004 & 0.193 & 0.061 \\
\hline$P$ & 0.642 & 0.617 & 0.520 & 0.976 & 0.142 & 0.650 \\
\hline \multicolumn{7}{|c|}{ Depression score } \\
\hline$r$ & -0.415 & -0.217 & -0.256 & -0.129 & -0.113 & -0.279 \\
\hline$P$ & $<0.00 I^{*}$ & 0.100 & 0.053 & 0.328 & 0.392 & $0.034 *$ \\
\hline \multicolumn{7}{|c|}{ MSNQ } \\
\hline$r$ & -0.236 & -0.097 & -0.068 & -0.105 & 0.035 & -0.130 \\
\hline$P$ & 0.072 & 0.466 & 0.613 & 0.431 & 0.793 & 0.331 \\
\hline \multicolumn{7}{|c|}{ Fatigue } \\
\hline$r$ & -0.383 & -0.110 & -0.239 & -0.245 & -0.068 & -272 \\
\hline$P$ & $0.003^{*}$ & 0.408 & 0.071 & 0.062 & 0.609 & $0.039 *$ \\
\hline
\end{tabular}

Note: *Significant $P$-value corrected for multiple testing using the Bonferroni correction method $(\alpha / k$ : 0.05/6=0.008).

Abbreviations: VM, verbal memory; VSM, visuospatial memory; PS, processing speed; WM, working memory; VF, verbal fluency; GCS, global cognitive score; EDSS, Expanded Disability Status Scale; MSNQ, Multiple Sclerosis Neuropsychological Questionnaire.
Table 5 Questionnaires results

\begin{tabular}{ll}
\hline & MS patients $(\mathbf{n}=\mathbf{6 0})$ \\
\hline MSNQ & $15.4 \pm 10.23(0-47)$ \\
FSS & $3.4 \pm 1.82(1-10)$ \\
HADS & \\
$\quad$ Anxiety & $4.7 \pm 3.21(0-14)$ \\
$\quad$ Depression & $4.8 \pm 3.81(0-16)$ \\
\hline
\end{tabular}

Note: Results are expressed as mean \pm SD (min-max).

Abbreviations: MS, multiple sclerosis; MSNQ, Multiple Sclerosis Neuropsychological Questionnaire; FSS, Fatigue Severity Scale; HADS, Hospital Anxiety and Depression Scale; SD, standard deviation; min, minimum; max, maximum.

Severe cognitive impairment, with clear criteria for dementia (DSM-IV-TR), was found in four patients in the study sample ( $6.3 \%$ of the total). All these four patients had an obvious neuropsychological deficit, and they were unable to perform all the selected tests. Interviews with family members revealed significant functional impairment with an impact on activities of daily living. The mean age of the dementia patients was 71 years (60-78 years), and they obtained an EDSS score between 6.0 and 8.0 as well as had progressive forms of the disease.

The remaining nondementia patients formed a population with a low-moderate degree of disability $(<3.0$ on the EDSS), and most patients had an RRMS.

The patient group underperformed the (demographically equivalent) $\mathrm{CG}$ in most of the battery tests after controlling the results for age and schooling, suggesting an overall performance deficit.

The range of variability observed in the prevalence of cognitive impairment is possibly associated with the methodological differences between the studies such as the study design and setting, as well as the neuropsychological tests selected for the study. However, the biggest difference could be due to the consideration of the concept of cognitive impairment. For example, a performance $<1.0 \mathrm{SD}$ in one subtest may reflect the fluctuant nature of the disease and does not necessarily reflect the real pattern of cognitive impairment. The concept of cognitive impairment proposed by Amato et $\mathrm{al}^{12}$ as a failure in two or more BRB-N subtests with scores at least $1.5 \mathrm{SD}$ below the scores of $\mathrm{HC}$ is suggested for use here.

Using the aforementioned criterion for defining abnormality showed that $35.5 \%$ of the patients had impairment. Similar figures were found by Nogales-Gaete et a ${ }^{39}$ with a sample of Chilean patients with RRMS, but the finding here differs from other studies conducted in Oslo (Norway) and the UK and from another study in Spanish speaking patients in Argentina, ${ }^{40}$ where the prevalence of cognitive impairment was $>45 \%$. 
The pattern of involvement found suggests that working memory and verbal memory are the most affected areas. Physical disability has been associated with cognitive impairment in some studies, ${ }^{6,14}$ while others found no such association. ${ }^{41}$ In the present work, a negative correlation between EDSS score and verbal memory, processing speed, and a global cognitive index was found. The association was strongest for processing speed. These data could indicate that deficit in processing speed might be a good predictor of disease progression and, therefore, constitutes a key deficit in time monitoring. However, the involvement of other domains such as working memory or verbal fluency could start in any stage of the disease and does not evolve in parallel with other symptoms of this disease.

MSNQ-Self report (as opposed to MSNQ-Informant report) is not considered a sensitive screen for neuropsychological impairment in MS, and indeed, no correlation with this test was found here, suggesting that it is not an effective screening method for detecting cognitive impairment.

Depressive symptoms were less (present in $33.3 \%$ of the sample) than those found in the literature, ${ }^{42}$ and higher scores on the depression scale were correlated with poorer performance in verbal memory. This finding has been associated with the mineralocorticoid expression in the brain that diminishes during depression, especially in the hippocampus and the prefrontal cortex, which are critical brain areas for memory. ${ }^{43}$ Anxiety (present in $18.3 \%$ of patients) was not associated with cognitive performance.

Fatigue was present in $36.7 \%$ of the sample and only correlated with verbal memory. This confirms findings of another study that has linked cognitive functioning with the state of fatigue in patients with $\mathrm{MS}^{44}$ and more specifically with memory. 45

As a limitation of the study, the use of a more extensive evaluation protocol could provide more information about the true neuropsychological profile of studied patients.

\section{Conclusion}

At least $35 \%$ of the study population had mild-to-moderate cognitive impairment relative to a control sample. The results of this study confirm the high prevalence of cognitive impairment in an MS cohort. The most affected domains were working memory and verbal memory. Working memory, verbal memory, and verbal fluency did not deteriorate as the disease progressed, and therefore could not be used as a means of monitoring it. Cognitive decline was frequently related to clinical variables (disease duration and EDSS score). Verbal memory was associated with depression and fatigue, but none of the cognitive domains showed a strong correlation with anxiety and subjective perception of cognitive impairment.

\section{Acknowledgment}

We would like to thank Patrick Dennis for his help in revising English of the manuscript.

\section{Disclosure}

The authors report no conflicts of interest in this work.

\section{References}

1. Cavallo M, Cavanna AE, Harciarek M, Johnston H, Ostacoli L, Angilletta C. 'Keep up the good work'! A case study of the effects of a specific cognitive training in Alzheimer's disease. Neurocase. 2013; 19(6):542-552.

2. Borghi M, Cavallo M, Carletto S, et al. Presence and significant determinants of cognitive impairment in a large sample of patients with multiple sclerosis. PLoS One. 2013;8(7):e69820.

3. Ding W, Ding L-J, Li F-F, Han Y, Mu L. Neurodegeneration and cognition in Parkinson's disease: a review. Eur Rev Med Pharmacol Sci. 2015; 19(12):2275-2281

4. Ransome MI, Renoir T, Hannan AJ. Hippocampal neurogenesis, cognitive deficits and affective disorder in Huntington's disease. Neural Plast. 2012;2012:874387.

5. Peyser JM, Rao SM, LaRocca NG, Kaplan E. Guidelines for neuropsychological research in multiple sclerosis. Arch Neurol. 1990;47(1): 94-97.

6. Rao SM, Leo GJ, Bernardin L, Unverzagt F. Cognitive dysfunction in multiple sclerosis. I. Frequency, patterns, and prediction. Neurology. 1991;41(5):685-691.

7. Chiaravalloti ND, DeLuca J. Cognitive impairment in multiple sclerosis. Lancet Neurol. 2008;7(12):1139-1151.

8. Calabrese P. Neuropsychology of multiple sclerosis - an overview. J Neurol. 2006;253(suppl 1):I10-I15.

9. Banati M, Sandor J, Mike A, et al. Social cognition and theory of mind in patients with relapsing-remitting multiple sclerosis. Eur J Neurol. 2010;17(3):426-433.

10. Huijbregts SCJ, Kalkers NF, de Sonneville LMJ, de Groot V, Reuling IEW, Polman $\mathrm{CH}$. Differences in cognitive impairment of relapsing remitting, secondary, and primary progressive MS. Neurology. 2004; 63(2):335-339.

11. Ruet A, Deloire M, Charré-Morin J,HamelD, Brochet B. Cognitive impairment differs between primary progressive and relapsing-remitting MS. Neurology. 2013;80(16):1501-1508.

12. Amato MP, Portaccio E, Goretti B, et al. Relevance of cognitive deterioration in early relapsing-remitting MS: a 3-year follow-up study. Mult Scler. 2010;16(12):1474-1482.

13. Reuter F, Zaaraoui W, Crespy L, et al; TuSCIMS Study Group. Frequency of cognitive impairment dramatically increases during the first 5 years of multiple sclerosis. J Neurol Neurosurg Psychiatry. 2011;82(10): $1157-1159$

14. Amato MP, Ponziani G, Siracusa G, Sorbi S. Cognitive dysfunction in early-onset multiple sclerosis: a reappraisal after 10 years. Arch Neurol. 2001;58(10):1602-1606.

15. Krupp LB, Elkins LE. Fatigue and declines in cognitive functioning in multiple sclerosis. Neurology. 2000;55(7):934-939.

16. Langdon DW. Cognition in multiple sclerosis. Curr Opin Neurol.2011; 24(3):244-249.

17. Schiavolin S, Leonardi M, Giovannetti AM, et al. Factors related to difficulties with employment in patients with multiple sclerosis: a review of 2002-2011 literature. Int J Rehabil Res. 2013;36(2):105-111. 
18. Bobholz JA, Rao SM. Cognitive dysfunction in multiple sclerosis: a review of recent developments. Curr Opin Neurol. 2003;16(3): 283-288.

19. Sundgren M, Maurex L, Wahlin Å, Piehl F, Brismar T. Cognitive impairment has a strong relation to nonsomatic symptoms of depression in relapsing-remitting multiple sclerosis. Arch Clin Neuropsychol. 2013;28(2):144-155.

20. Goretti B, Portaccio E, Zipoli V, Razzolini L, Amato MP. Coping strategies, cognitive impairment, psychological variables and their relationship with quality of life in multiple sclerosis. Neurol Sci. 2010; 31(suppl 2):S227-S230.

21. Morrow SA, Rosehart H, Pantazopoulos K. Anxiety and depressive symptoms are associated with worse performance on objective cognitive tests in MS. J Neuropsychiatry Clin Neurosci. 2015;28(2):118-123.

22. Garcia JR, Rodriguez S, Sosa Henriquez M, et al. Prevalence of multiple sclerosis in Lanzarote (Canary Islands). Neurology. 1989;39(2 pt 1): 265-267.

23. Eguía del Río P, Suárez Cuervo A, Hadjigeorgiou I, Pineda Pineda G, Pérez Martín MY, González Platas M. Baja prevalencia e incidencia de esclerosis múltiple en la isla de Lanzarote [Low prevalence and incidence of multiple sclerosis on the island of Lanzarote] [Abstract]. Neurología. 2013;28:217. Spanish.

24. Hernández MA. Epidemiology of multiple sclerosis in the Canary Islands (Spain): a study on the island of La Palma. J Neurol. 2002;249(10): 1378-1381.

25. Aladro Y, Alemany MJ, Pérez-Vieitez MC, et al. Prevalence and incidence of multiple sclerosis in Las Palmas, Canary Islands, Spain. Neuroepidemiology. 2005;24(1-2):70-75.

26. WMA [webpage on the Internet]. WMA Declaration of Helsinki Ethical Principles for Medical Research Involving Human Subjects [Internet]; 2013 [cited January 9, 2016]. Available from: http://www. wma.net/es/30publications/10policies/b3/. Accessed April 23, 2016.

27. Polman $\mathrm{CH}$, Reingold SC, Edan G, et al. Diagnostic criteria for multiple sclerosis: 2005 revisions to the 'McDonald Criteria'. Ann Neurol. 2005;58(6):840-846.

28. Buschke H, Fuld PA. Evaluating storage, retention, and retrieval in disordered memory and learning. Neurology. 1974;24(11):1019-1025.

29. Barbizet J, Cany E. Clinical and psychometrical study of a patient with memory disturbances. Int J Neurol. 1968;7(1):44-54.

30. Smith A. Symbol Digit Modalities Test: Manual. Torrance, CA: Western Psychological Corporation; 2002.

31. Gronwall DM. Paced auditory serial-addition task: a measure of recovery from concussion. Percept Mot Skills. 1977;44(2):367-373.

32. Spreen O, Benton AL. Neurosensory Center Comprehensive Examination for Aphasia: Manual of Directions. Victoria, BC: Neuropsychology Laboratory, University of Victoria; 1969.
33. Benedict RH, Munschauer F, Linn R, et al. Screening for multiple sclerosis cognitive impairment using a self-administered 15-item questionnaire. Mult Scler. 2003;9(1):95-101.

34. Zigmond AS, Snaith RP. The hospital anxiety and depression scale. Acta Psychiatr Scand. 1983;67(6):361-370.

35. Krupp LB, LaRocca NG, Muir-Nash J, Steinberg AD. The fatigue severity scale. Application to patients with multiple sclerosis and systemic lupus erythematosus. Arch Neurol. 1989;46(10):1121-1123.

36. American Psychiatric Association. Diagnostic and Statistical Manual of Mental Disorders: DSM-IV-TR. 4th ed Reviewed. Washington, DC: APA; 2000.

37. Pierrot-Deseilligny $\mathrm{C}$, Souberbielle J-C. Is hypovitaminosis D one of the environmental risk factors for multiple sclerosis? Brain. 2010;133(pt 7): 1869-1888.

38. Fitzgerald KC, Munger KL, Köchert K, et al. Association of vitamin D levels with multiple sclerosis activity and progression in patients receiving interferon beta-1b. JAMA Neurol. 2015;72(12):1458-1465.

39. Nogales-Gaete J, Aracena R, Díaz V, et al. Evaluación neuropsicológica en 129 pacientes chilenos con esclerosis múltiple recurrente remitente previo a inicio de fármacos inmunomoduladores [Neuropsychological assessment of patients with relapsing remitting multiple sclerosis prior to the use of immunomodulatory drugs]. Rev Méd Chil. 2012;140(11): 1437-1444. Spanish.

40. Cáceres F, Vanotti S, Rao S; RECONEM Workgroup. Epidemiological characteristics of cognitive impairment of multiple sclerosis patients in a Latin American country. J Clin Exp Neuropsychol. 2011;33(10): 1094-1098.

41. Beatty WW, Goodkin DE, Hertsgaard D, Monson N. Clinical and demographic predictors of cognitive performance in multiple sclerosis. Do diagnostic type, disease duration, and disability matter? Arch Neurol. 1990;47(3):305-308.

42. Siegert RJ, Abernethy DA. Depression in multiple sclerosis: a review. J Neurol Neurosurg Psychiatry. 2005;76(4):469-475.

43. Otte C, Wingenfeld K, Kuehl LK, et al. Cognitive function in older adults with major depression: effects of mineralocorticoid receptor stimulation. J Psychiatr Res. 2015;69:120-125.

44. Cameron MH, Peterson V, Boudreau EA, et al. Fatigue is associated with poor sleep in people with multiple sclerosis and cognitive impairment. Mult Scler Int. 2014;2014:872732.

45. Jougleux-Vie C, Duhin E, Deken V, Outteryck O, Vermersch P, Zéphir H. Does fatigue complaint reflect memory impairment in multiple sclerosis? Mult Scler Int. 2014;2014:692468.
Neuropsychiatric Disease and Treatment

\section{Publish your work in this journal}

Neuropsychiatric Disease and Treatment is an international, peerreviewed journal of clinical therapeutics and pharmacology focusing on concise rapid reporting of clinical or pre-clinical studies on a range of neuropsychiatric and neurological disorders. This journa is indexed on PubMed Central, the 'PsycINFO' database and CAS,

\section{Dovepress}

and is the official journal of The International Neuropsychiatric Association (INA). The manuscript management system is completely online and includes a very quick and fair peer-review system, which is all easy to use. Visit http://www.dovepress.com/testimonials.php to read real quotes from published authors. 